PROCEEDINGS OF THE

AMERICAN MATHEMATICAL SOCIETY

Volume 127, Number 1, January 1999, Pages 145-154

S 0002-9939(99)04845-5

\title{
GENERALIZED POWER MEANS AND INTERPOLATING INEQUALITIES
}

\author{
HSU-TUNG KU, MEI-CHIN KU, AND XIN-MIN ZHANG
}

(Communicated by Frederick W. Gehring)

\begin{abstract}
In this paper, we introduce a multi-parameter family of generalized power means, and use their special properties to provide a new method of interpolating inequalities. We give a different refinement of an inequality of Ky Fan as a particular application of our method.
\end{abstract}

\section{INTRODUCTION}

Interpolation of inequalities extends known inequalities in a systematic way by inserting inequalities between the extremes, or introducing parameters into some functions with special values at those extremes. For example, the $\lambda$-method by D. S. Mitrinović and P. M. Vasić, and the functional equation approach by S. Iwamoto are discussed in [12]. Due to numerous references on inequalities and the large variety of methods, we shall recommend to the interested reader the excellent books $[3,4,5,11,12,13]$ and further relevant articles therein.

Let $\mathbf{a}=\left(a_{1}, \cdots, a_{n}\right) \in \mathbf{R}_{+}^{n}$, where $\mathbf{R}_{+}$denotes the set of all positive real numbers, and

$$
A_{n}(\mathbf{a})=\frac{1}{n} \sum_{i=1}^{n} a_{i} \quad \text { and } \quad G_{n}(\mathbf{a})=\left[\prod_{i=1}^{n} a_{i}\right]^{\frac{1}{n}}
$$

denote the arithmetic mean and the geometric mean of $a_{1}, \cdots, a_{n}$, respectively. One of the most important inequalities, perhaps a keystone of the theory of inequalities, is the arithmetic mean-geometric mean inequality ([3, p. 3]), that is,

$$
G_{n}(\mathbf{a}) \leq A_{n}(\mathbf{a})
$$

with equality if and only if $a_{1}=a_{2}=\cdots=a_{n}$.

Furthermore, let $H_{n}(\mathbf{a})=n\left[\sum_{i=1}^{n} 1 / a_{i}\right]^{-1}$ be the harmonic mean of $a_{1}, \cdots, a_{n}$. It is also known that $([12])$

$$
H_{n}(\mathbf{a}) \leq G_{n}(\mathbf{a}) \leq A_{n}(\mathbf{a}),
$$

where, in either case, equality holds if and only if $a_{1}=a_{2}=\cdots=a_{n}$.

Received by the editors May 2, 1997.

1991 Mathematics Subject Classification. Primary 26B25, 26D05, 26D10.

Key words and phrases. Arithmetic mean, geometric mean, harmonic mean, symmetric mean, generalized power mean, interpolating inequalities. 
A simple example of interpolating inequality (2) is to introduce the so-called symmetric means. For $\mathbf{a}=\left(a_{1}, \cdots, a_{n}\right) \in \mathbf{R}_{+}^{n}$, denote $1 / \mathbf{a}=\left(1 / a_{1}, \cdots, 1 / a_{n}\right)$, and let

$$
\sigma_{r}(\mathbf{a})=\sum_{1 \leq i_{1}<\cdots<i_{r} \leq n} a_{i_{1}} \cdots a_{i_{r}},
$$

be the $r$ th elementary symmetric function of $a_{1}, \cdots, a_{n}$; the summation is taken over all possible permutations of $\left\{i_{1}, \cdots, i_{r}\right\}, r=1, \cdots, n$. Define the $r$ th symmetric mean of a as

$$
P_{n}^{[r]}(\mathbf{a})=\left\{\left[\begin{array}{l}
n \\
r
\end{array}\right]^{-1} \sigma_{r}(\mathbf{a})\right\}^{1 / r}, \quad r=1,2, \cdots, n .
$$

Moreover we define

$$
P_{n}^{[-r]}(\mathbf{a})=\left\{P_{n}^{[r]}\left(\frac{1}{\mathbf{a}}\right)\right\}^{-1}, \quad r=1,2, \cdots, n .
$$

It is well-known that $([12,13])$ :

$$
\begin{aligned}
H_{n}(\mathbf{a}) & =P_{n}^{[-1]}(\mathbf{a}) \leq \cdots \leq P_{n}^{[-n+1]}(\mathbf{a}) \leq P_{n}^{[-n]}(\mathbf{a})=G_{n}(\mathbf{a}) \\
& =P_{n}^{[n]}(\mathbf{a}) \leq \cdots \leq P_{n}^{[2]}(\mathbf{a}) \leq P_{n}^{[1]}(\mathbf{a})=A_{n}(\mathbf{a})
\end{aligned}
$$

with equality if and only if $a_{1}=a_{2}=\cdots=a_{n}$.

In the chain of inequalities above, the symmetric means $P_{n}^{[r]}(\mathbf{a}), r= \pm 1, \cdots, \pm n$, can be viewed as a family of $2 n-1$ means with the arithmetic mean $A_{n}(\mathbf{a})$ as the largest member and the harmonic mean $H_{n}(\mathbf{a})$ as the smallest. A direct calculation shows that

$$
\left\{G_{n}(\mathbf{a})\right\}^{n}=H_{n}(\mathbf{a})\left\{P_{n}^{[n-1]}(\mathbf{a})\right\}^{n-1} \quad \text { and } \quad\left\{G_{n}(\mathbf{a})\right\}^{n}=A_{n}(\mathbf{a})\left\{P_{n}^{[-n+1]}(\mathbf{a})\right\}^{n-1} .
$$

Motivated by different considerations, there has been a great deal of effort devoted to the development of a continuous family of means with diverse applications. Some of the methods are quite elementary but appealing, and some are sophisticated (cf. $[9,18])$. For instance, if we define for $x$ real, $x \neq 0$,

$$
F(x)(\mathbf{a})=\left(\frac{a_{1}^{x}+a_{2}^{x}+\cdots+a_{n}^{x}}{n}\right)^{1 / x},
$$

then it can be verified that ([18]) $G_{n}(\mathbf{a})=\lim _{x \longrightarrow 0} F(x)(\mathbf{a}), F(x)(\mathbf{a})$ is a monotonic increasing function, and it is continuous everywhere if we define $F(0)(\mathbf{a})=G_{n}(\mathbf{a})$. It is clear that

$$
F(-1)(\mathbf{a})=H_{n}(\mathbf{a}), \quad F(0)(\mathbf{a})=G_{n}(\mathbf{a}), \quad F(1)(\mathbf{a})=A_{n}(\mathbf{a}),
$$

and $F(2)(\mathbf{a})=Q_{n}(\mathbf{a})$ is the root mean of $a_{1}, \cdots, a_{n}$. Moreover, denote by $M$ and $m$ the largest element and the smallest element of $a_{1}, a_{2}, \cdots, a_{n}$ respectively. Then from [18] we have

$$
\lim _{x \longrightarrow \infty} F(x)(\mathbf{a})=M \quad \text { and } \quad \lim _{x \longrightarrow-\infty} F(x)(\mathbf{a})=m .
$$

Obviously, $F(x)$ serves as a continuous family of means with particular values as those basic means. Recently, Yang and Wang have introduced different monotonic functions with special values as various means in an endeavour to generalize a famous inequality of Ky Fan ([20]). In the early 1980's, Leach and Sholander studied some multi-variable extended mean values via different functions which possess special properties ([9]). 
In the next section, we shall introduce a multi-parameter family of means that will be used to interpolate many useful inequalities including the one of Ky Fan.

\section{Generalized power means}

Definition 2.1. Let $\mathbf{a}=\left(a_{1}, \cdots, a_{n}\right), \mathbf{w}=\left(w_{1}, \cdots, w_{n}\right) \in \mathbf{R}_{+}^{n}$. Set $w_{+}=\sum_{i=1}^{n} w_{i}$. The $r$ th power mean of $\mathbf{a}$ with weight $\mathbf{w}, M_{n}^{[r]}(\mathbf{a} ; \mathbf{w})$ is defined by

$$
M_{n}^{[r]}(\mathbf{a} ; \mathbf{w})= \begin{cases}{\left[\prod_{i=1}^{n} a_{i}^{w_{i}}\right]^{\frac{1}{w_{+}}}} & \text {if } r=0, \\ {\left[\frac{\sum_{i=1}^{n} w_{i} a_{i}^{r}}{w_{+}}\right]^{\frac{1}{r}}} & \text { if } r \neq 0 .\end{cases}
$$

More generally, define means due to Gini and Bonferroin (cf. [4, p. 189]) $B_{n}^{r, t}(\mathbf{a} ; \mathbf{w})$, $r \geq t \geq 0$, as follows:

$$
B_{n}^{r, t}(\mathbf{a} ; \mathbf{w})= \begin{cases}{\left[\prod_{i=1}^{n} a_{i}^{w_{i}} a_{i}^{r}\right]^{\frac{1}{\sum_{i=1}^{n} w_{i} a_{i}^{r}}}} & \text { if } r=t, \\ {\left[\frac{\sum_{i=1}^{n} w_{i} a_{i}^{r}}{\sum_{i=1}^{n} w_{i} a_{i}^{t}}\right]^{\frac{1}{r-t}}} & \text { if } r>t .\end{cases}
$$

Then $B_{n}^{r, 0}(\mathbf{a} ; \mathbf{w})=M_{n}^{[r]}(\mathbf{a} ; \mathbf{w})$. The counterharmonic mean $H_{n}^{[r]}(\mathbf{a} ; \mathbf{w}), r \geq 1$, is thereby defined by $H_{n}^{[r]}(\mathbf{a} ; \mathbf{w})=B_{n}^{r, r-1}(\mathbf{a} ; \mathbf{w})$.

The most important property of the power means is perhaps the following inequality.

Theorem $2.2\left(\left[4\right.\right.$, p. 159]). Let $-\infty<t<r<+\infty$. Then for $\mathbf{a}, \mathbf{w} \in \mathbf{R}_{+}^{n}$,

$$
M_{n}^{[t]}(\mathbf{a} ; \mathbf{w}) \leq M_{n}^{[r]}(\mathbf{a} ; \mathbf{w}),
$$

with equality if and only if $a_{1}=a_{2}=\cdots=a_{n}$.

Remark. When $\mathbf{w}=(1 / n, \cdots, 1 / n)$, then $M_{n}^{[1]}(\mathbf{a} ; \mathbf{w})=A_{n}(\mathbf{a}), M_{n}^{[0]}(\mathbf{a} ; \mathbf{w})=$ $G_{n}(\mathbf{a})$, and $M_{n}^{[-1]}(\mathbf{a} ; \mathbf{w})=H_{n}(\mathbf{a})$. Theorem 2.2 is simply another generalization of the classical inequality (2). With $\mathbf{a}$ and $\mathbf{w}$ fixed, the power mean can be viewed as a monotonic function of $r$.

Now, let $\mathbf{Q}_{n} \subset \mathbf{R}_{+}^{n},(n \geq 2)$, be a non-empty subset, and $\mathbf{f}=\left(f_{1}, f_{2}, \cdots f_{m}\right)$, where $f_{i}: \mathbf{Q}_{n} \longrightarrow \mathbf{R}_{+}, 1 \leq i \leq m$, are distinct functions. Let $w_{i}>0,1 \leq i \leq m$, and $\triangle(\mathbf{w})=\triangle\left(w_{1}, \cdots, w_{m}\right)$ be the $(m-1)$-simplex in $\mathbf{R}^{m}$ with vertices

$\mathbf{W}_{i}=\left(0, \cdots, 0,1 / w_{i}, 0, \cdots, 0\right) \quad$ where $1 / w_{i}$ is the $i$ th coordinate, $i=1, \cdots, m$.

Thus, if $\mathbf{x}=\left(x_{1}, \cdots, x_{m}\right) \in \triangle(\mathbf{w})$, then $\sum_{i=1}^{m} w_{i} x_{i}=1$.

Definition 2.3. For $\mathbf{x} \in \triangle(\mathbf{w}), \mathbf{a} \in \mathbf{Q}_{n}$ and $r \geq 0$, define the generalized power mean $L_{n, m}^{[r]}[\mathbf{f} ; \mathbf{x} ; \mathbf{w}](\mathbf{a})$ by

$$
L_{n, m}^{[r]}[\mathbf{f} ; \mathbf{x} ; \mathbf{w}](\mathbf{a})= \begin{cases}\prod_{i=1}^{m}\left\{f_{i}(\mathbf{a})\right\}^{w_{i} x_{i}} & \text { if } r=0, \\ \left\{\sum_{i=1}^{m} w_{i} x_{i}\left[f_{i}(\mathbf{a})\right]^{r}\right\}^{\frac{1}{r}} & \text { if } r>0 .\end{cases}
$$

Notice that if we define $f_{i}(\mathbf{a})=a_{i}$ for $\mathbf{a} \in \mathbf{R}_{+}^{n}$, and choose $x_{i}=1 / w_{+}, 1 \leq i \leq n$, then

$$
L_{n, m}^{[r]}[\mathbf{f} ; \mathbf{x} ; \mathbf{w}](\mathbf{a})=M_{n}^{[r]}(\mathbf{a}, \mathbf{w}), \quad r \geq 0 .
$$

The generalized power means are also well-defined for $r<0$. For simplicity we will consider only the case when $r \geq 0$. In order to have an inequality comparable to Theorem 2.2, we now introduce an index dominant relation $\succ$ on $\triangle(\mathbf{w})$ as follows. 
For $\mathbf{x}, \mathbf{x}^{\prime} \in \triangle(\mathbf{w}), \mathbf{w} \in \mathbf{R}_{+}^{n}$, define $\mathbf{x} \succ \mathbf{x}^{\prime}$ if $\mathbf{x}=\mathbf{x}^{\prime}$, or there exists an integer $k, 1 \leq k<m$ such that the following conditions are satisfied:

$x_{i} \geq x_{i}^{\prime}$ for $1 \leq i \leq k ; \quad x_{k+1}<x_{k+1}^{\prime} ; x_{i} \leq x_{i}^{\prime}$ for $k+2 \leq i \leq m$, if $k+2 \leq m$.

Furthermore, for $\mathbf{a} \in \mathbf{Q}_{n}, \mathbf{x} \succ \mathbf{x}^{\prime}$, and $\mathbf{x} \neq \mathbf{x}^{\prime}$, we give the definition of the statement $\mathrm{EQ}\left(\mathbf{f} ; \mathbf{x} \succ \mathbf{x}^{\prime} ; \mathbf{a}\right)$ as follows:

$$
\operatorname{EQ}\left(\mathbf{f} ; \mathbf{x} \succ \mathbf{x}^{\prime} ; \mathbf{a}\right): \quad \sum_{i=1, i \neq k, x_{i} \neq x_{i}^{\prime}}^{m}\left[f_{i}(\mathbf{a})-f_{k}(\mathbf{a})\right]^{2}=0 \Longleftrightarrow a_{1}=a_{2}=\cdots=a_{n} .
$$

For instance, let $\mathbf{f}$ satisfy the following condition: for any pair $(i, j), i \neq j, \mathbf{a} \in \mathbf{Q}_{n}$,

$$
f_{i}(\mathbf{a})=f_{j}(\mathbf{a}) \Longleftrightarrow a_{1}=a_{2}=\cdots=a_{n} .
$$

Then $\mathrm{EQ}\left(\mathbf{f} ; \mathbf{x} \succ \mathbf{x}^{\prime} ; \mathbf{a}\right)$ holds for any $\mathbf{x}, \mathbf{x}^{\prime}, \mathbf{x} \succ \mathbf{x}^{\prime}$, and $\mathbf{x} \neq \mathbf{x}^{\prime}$. Some non-trivial examples are given below.

Example 2.4. Let $\mathbf{Q}_{n}=\mathbf{R}_{+}^{n}$. The following functions satisfy (4).

(a) $f_{i}(\mathbf{a})=P_{n}^{\left[r_{i}\right]}(\mathbf{a}), \quad 1 \leq r_{1}<r_{2}<\cdots<r_{m} \leq n$.

(b) $f_{i}(\mathbf{a})=P_{n}^{\left[-r_{i}\right]}(\mathbf{a}), \quad 1 \leq r_{1}<r_{2}<\cdots<r_{m} \leq n$.

(c) $f_{i}(\mathbf{a})=A_{n}^{\left[r_{i}\right]}(\mathbf{a}), \quad 1 \leq r_{1}<r_{2}<\cdots<r_{m} \leq n$, where $A_{n}^{[r]}(\mathbf{a})$ is defined as

$$
A_{n}^{[r]}(\mathbf{a})=\left\{A_{n}(\mathbf{a})\right\}^{\frac{n-r}{r(n-1)}}\left\{G_{n}(\mathbf{a})\right\}^{\frac{n(r-1)}{r(n-1)}} .
$$

(d) $f_{i}(\mathbf{a})=M_{n}^{\left[r_{i}\right]}(\mathbf{a} ; \mathbf{w}), 0 \leq r_{1}<r_{2}<\cdots<r_{m}$.

We now present our main result as follows.

Theorem 2.5. (a) If $0 \leq t<r, \mathbf{x} \in \triangle(\mathbf{w})$,

$$
L_{n, m}^{[t]}[\mathbf{f} ; \mathbf{x} ; \mathbf{w}](\mathbf{a}) \leq L_{n, m}^{[r]}[\mathbf{f} ; \mathbf{x} ; \mathbf{w}](\mathbf{a}), \quad \mathbf{a} \in \mathbf{Q}_{n} .
$$

Equality holds if and only if $a_{1}=a_{2}=\cdots=a_{n}$.

(b) Suppose $\mathbf{x} \succ \mathbf{x}^{\prime} ; \mathbf{x}, \mathbf{x}^{\prime} \in \triangle(\mathbf{w})$ and $\mathbf{f}$ satisfies

$$
f_{1}(\mathbf{a}) \geq f_{2}(\mathbf{a}) \geq \cdots \geq f_{m}(\mathbf{a}), \quad \mathbf{a} \in \mathbf{Q}_{n} .
$$

Then for any $r \geq 0$,

$$
L_{n, m}^{[r]}[\mathbf{f} ; \mathbf{x} ; \mathbf{w}](\mathbf{a}) \geq L_{n, m}^{[r]}\left[\mathbf{f} ; \mathbf{x}^{\prime} ; \mathbf{w}\right](\mathbf{a}), \quad \mathbf{a} \in \mathbf{Q}_{n} .
$$

If $\mathbf{x} \neq \mathbf{x}^{\prime}$, and $\mathbf{f}$ satisfies the condition $E Q\left(\mathbf{f} ; \mathbf{x} \succ \mathbf{x}^{\prime} ; \mathbf{a}\right)$, then equality holds if and only if $a_{1}=a_{2}=\cdots=a_{n}$.

Proof. (a) The inequality follows from Theorem 2.2 because

$$
L_{n, m}^{[r]}[\mathbf{f} ; \mathbf{x} ; \mathbf{w}](\mathbf{a})=M_{n}^{[r]}\left(\left(f_{1}(\mathbf{a}), \cdots, f_{m}(\mathbf{a})\right),\left(w_{1} x_{1}, \cdots, w_{m} x_{m}\right)\right) .
$$

(b) For $\mathbf{a} \in \mathbf{Q}_{n}$, choose $\beta>0$ so that $\beta f_{m}(\mathbf{a}) \geq 1$. First, let us consider the case $r=0$. For $1 \leq i \leq k$,

$$
\left(\beta f_{i}\right)^{w_{i} x_{i}}(\mathbf{a})=\left\{\beta f_{i}(\mathbf{a})\right\}^{w_{i} x_{i}}=\left(\beta f_{i}\right)^{w_{i} x_{i}^{\prime}}(\mathbf{a})\left(\beta f_{i}\right)^{w_{i}\left(x_{i}-x_{i}^{\prime}\right)}(\mathbf{a}),
$$

hence by $(5)$, we have

$$
\left(\beta f_{i}\right)^{w_{i} x_{i}}(\mathbf{a}) \geq\left(\beta f_{i}\right)^{w_{i} x_{i}^{\prime}}(\mathbf{a})\left(\beta f_{k}\right)^{w_{i}\left(x_{i}-x_{i}^{\prime}\right)}(\mathbf{a}) .
$$


As $\mathbf{x}, \mathbf{x}^{\prime} \in \triangle(\mathbf{w}), \sum_{i=1}^{m} w_{i} x_{i}=\sum_{i=1}^{m} w_{i} x_{i}^{\prime}=1$, hence

$$
\sum_{i=1}^{k} w_{i}\left(x_{i}-x_{i}^{\prime}\right)=\sum_{i=k+1}^{m} w_{i}\left(x_{i}^{\prime}-x_{i}\right) .
$$

It follows from (5), (6) and (7) that

$$
\begin{aligned}
\beta L_{n, m}^{[r]}[\mathbf{f} ; \mathbf{x} ; \mathbf{w}](\mathbf{a}) & \\
= & \prod_{i=1}^{k}\left(\beta f_{i}\right)^{w_{i} x_{i}}(\mathbf{a}) \prod_{i=k+1}^{m}\left(\beta f_{i}\right)^{w_{i} x_{i}}(\mathbf{a}) \\
\geq & \prod_{i=1}^{k}\left(\beta f_{i}\right)^{w_{i} x_{i}^{\prime}}(\mathbf{a})\left(\beta f_{k}\right)^{\sum_{i=1}^{k} w_{i}\left(x_{i}-x_{i}^{\prime}\right)}(\mathbf{a}) \prod_{i=k+1}^{m}\left(\beta f_{i}\right)^{w_{i} x_{i}}(\mathbf{a}) \\
= & \prod_{i=1}^{k}\left(\beta f_{i}\right)^{w_{i} x_{i}^{\prime}}(\mathbf{a})\left(\beta f_{k}\right)^{\sum_{i=k+1}^{m} w_{i}\left(x_{i}^{\prime}-x_{i}\right)}(\mathbf{a}) \prod_{i=k+1}^{m}\left(\beta f_{i}\right)^{w_{i} x_{i}}(\mathbf{a}) \\
\geq & \prod_{i=1}^{k}\left(\beta f_{i}\right)^{w_{i} x_{i}^{\prime}}(\mathbf{a}) \prod_{i=k+1}^{m}\left(\beta f_{i}\right)^{w_{i}\left(x_{i}^{\prime}-x_{i}\right)}(\mathbf{a}) \prod_{i=k+1}^{m}\left(\beta f_{i}\right)^{w_{i} x_{i}}(\mathbf{a}) \\
= & \beta \prod_{i=1}^{m} f_{i}^{w_{i} x_{i}^{\prime}}(\mathbf{a}) \\
& =\beta L_{n, m}^{[r]}\left[\mathbf{f} ; \mathbf{x}^{\prime} ; \mathbf{w}\right](\mathbf{a}) .
\end{aligned}
$$

Equality holds if and only if for $i \neq k, 1 \leq i \leq m$, we have

$$
\left(\beta f_{i}\right)^{w_{i}\left(x_{i}-x_{i}^{\prime}\right)}(\mathbf{a})=\left(\beta f_{k}\right)^{w_{i}\left(x_{i}-x_{i}^{\prime}\right)}(\mathbf{a}) .
$$

Since $\mathbf{x} \succ \mathbf{x}^{\prime}$, there exists at least one $i$ such that $x_{i} \neq x_{i}^{\prime}$, hence (8) implies that $f_{i}(\mathbf{a})=f_{k}(\mathbf{a})$ if $x_{i} \neq x_{i}^{\prime}$. By the equality condition $\mathrm{EQ}\left(\mathbf{f} ; \mathbf{x} \succ \mathbf{x}^{\prime} ; \mathbf{a}\right)$ we have $a_{1}=a_{2}=\cdots=a_{n}$.

For $r>0$, the proof is similar.

$$
\begin{aligned}
\beta^{r}\{ & \left.L_{n, m}^{[r]}[\mathbf{f} ; \mathbf{x} ; \mathbf{w}](\mathbf{a})\right\}^{r} \\
& \geq \sum_{i=1}^{k} w_{i} x_{i}^{\prime}\left(\beta f_{i}\right)^{r}(\mathbf{a})+\sum_{i=1}^{k} w_{i}\left(x_{i}-x_{i}^{\prime}\right)\left(\beta f_{k}\right)^{r}(\mathbf{a})+\sum_{i=k+1}^{m} w_{i} x_{i}\left(\beta f_{i}\right)^{r}(\mathbf{a}) \\
& \geq \sum_{i=1}^{k} w_{i} x_{i}^{\prime}\left(\beta f_{i}\right)^{r}(\mathbf{a})+\sum_{i=k+1}^{m} w_{i}\left(x_{i}^{\prime}-x_{i}\right)\left(\beta f_{k}\right)^{r}(\mathbf{a})+\sum_{i=k+1}^{m} w_{i} x_{i}\left(\beta f_{i}\right)^{r}(\mathbf{a}) \\
& \geq \sum_{i=1}^{k} w_{i} x_{i}^{\prime}\left(\beta f_{i}\right)^{r}(\mathbf{a})+\sum_{i=k+1}^{m} w_{i}\left(x_{i}^{\prime}-x_{i}\right)\left(\beta f_{i}\right)^{r}(\mathbf{a})+\sum_{i=k+1}^{m} w_{i} x_{i}\left(\beta f_{i}\right)^{r}(\mathbf{a}) \\
& =\beta^{r} \sum_{i=1}^{m} w_{i} x_{i}^{\prime} f_{i}^{r}(\mathbf{a}) \\
& =\beta^{r}\left\{L_{n, m}^{[r]}\left[\mathbf{f} ; \mathbf{x}^{\prime} ; \mathbf{w}\right](\mathbf{a})\right\}^{r} .
\end{aligned}
$$

Equality holds if and only if

$$
w_{i}\left(x_{i}-x_{i}^{\prime}\right) f_{i}^{r}(\mathbf{a})=w_{i}\left(x_{i}-x_{i}^{\prime}\right) f_{k}^{r}(\mathbf{a}), \quad i \neq k, \quad 1 \leq i \leq m .
$$


Again, we use the hypothesis $\operatorname{EQ}\left(\mathbf{f} ; \mathbf{x} \succ \mathbf{x}^{\prime} ; \mathbf{a}\right)$ concluding that $a_{1}=a_{2}=\cdots=$ $a_{n}$.

Remarks 2.6. (i) Theorem 2.5 (a) becomes Theorem 2.2 if we choose $f_{i}(\mathbf{a})=a_{i}$ for $i=1, \cdots, n$. (ii) We can apply Theorem 2.5 (b) to the functions in Example 2.4. The following special case of Theorem 2.5 (b) was proved in [7]: $m=3, w_{1}=1$, $w_{2}=n-1, w_{3}=n / 2, n \geq 3, f_{1}(\mathbf{a})=A_{n}(\mathbf{a}), f_{2}(\mathbf{a})=\left\{\left[A_{n}(\mathbf{a})\right]^{n-2}\left[G_{n}(\mathbf{a})\right]^{n}\right\}^{\frac{1}{2(n-1)}}$, and $f_{3}(\mathbf{a})=G_{n}(\mathbf{a})$.

Theorem 2.5 (b) generalizes many important inequalities (more applications will be given in the next section). For instance, let us consider some inequalities involving $H_{n}^{[r]}(\mathbf{a} ; \mathbf{w})$, or $B_{n}^{r, t}(\mathbf{a} ; \mathbf{w})$ below. For $\mathbf{a} \in \mathbf{R}_{+}^{n}$, we can assume that $a_{1} \geq a_{2} \geq \cdots \geq a_{n}$ (by rearranging the subscripts). Choose $\beta>0$ so that $\beta a_{n} \geq 1$. Let $r>t \geq 0$. For $\mathbf{w} \in \mathbf{R}_{+}^{n}$, set

$$
\begin{gathered}
u=\sum_{i=1}^{n} w_{i} a_{i}^{r}, \quad v=\sum_{i=1}^{n} w_{i} a_{i}^{t}, \\
x_{j}=a_{j}^{r} / u, \quad \text { and } \quad x_{j}^{\prime}=a_{j}^{t} / v, \quad 1 \leq j \leq n .
\end{gathered}
$$

Notice that

$$
x_{j}=\frac{\left(\beta a_{j}\right)^{r}}{\sum_{i=1}^{n} w_{i}\left(\beta a_{i}\right)^{r}} \quad \text { and } \quad x_{j}^{\prime}=\frac{\left(\beta a_{j}\right)^{t}}{\sum_{i=1}^{n} w_{i}\left(\beta a_{i}\right)^{t}} .
$$

Set $c=\sum_{i=1}^{n} w_{i}\left(\beta a_{i}\right)^{r} / \sum_{i=1}^{n} w_{i}\left(\beta a_{i}\right)^{t}$, as $r>t$ and $\beta a_{n} \geq 1$, we have $c \geq 1$.

Lemma 2.7. Let $\mathbf{x}, \mathbf{x}^{\prime} \in \mathbf{R}_{+}^{n}$ be defined by (10). Then $\mathbf{x}, \mathbf{x}^{\prime} \in \triangle(\mathbf{w})$ and $\mathbf{x} \succ \mathbf{x}^{\prime}$.

Proof. It is clear that

$$
\sum_{i=1}^{n} w_{i} x_{i}=\sum_{i=1}^{n} w_{i} x_{i}^{\prime}=1 \text {. }
$$

Hence, $\mathbf{x}, \mathbf{x}^{\prime} \in \triangle(\mathbf{w})$. If $a_{1}=\cdots=a_{n}$, then $\mathbf{x}=\mathbf{x}^{\prime}$. Suppose now that not all $a_{j}$ 's are equal. Since $x_{j}^{\prime} / x_{j}=c\left(\beta a_{j}\right)^{t-r}$, we obtain

$$
x_{j}^{\prime} \leq x_{j}\left(\text { resp. } x_{j}^{\prime} \geq x_{j}\right) \Longleftrightarrow c \leq\left(\beta a_{j}\right)^{r-t}\left(\text { resp. } c \geq\left(\beta a_{j}\right)^{r-t}\right) .
$$

If $x_{j}^{\prime} \leq x_{j}$ (resp. $x_{j}^{\prime} \geq x_{j}$ ) for $1 \leq j \leq n$, then we shall have $a_{1}=a_{2}=\cdots=a_{n}$, and this is a contradiction. Clearly if $x_{j}^{\prime} \leq x_{j}$ for $1 \leq j \leq n$, then $x_{j}^{\prime}=x_{j}$ for $1 \leq j \leq n$ by (11), and so, $c=\left(\beta a_{j}\right)^{r-t}, 1 \leq j \leq n$ by (12). Thus, there exists a largest positive integer $k, k<n$, so that either

$$
\begin{gathered}
x_{k}^{\prime} \leq x_{k} \quad \text { and } \quad x_{k+1}^{\prime}>x_{k+1}, \quad \text { or } \\
x_{k}^{\prime} \geq x_{k} \quad \text { and } \quad x_{k+1}^{\prime}<x_{k+1} .
\end{gathered}
$$

Suppose (14) holds, then by (12)

$$
\left(\beta a_{k}\right)^{r-t} \leq c \quad \text { and } \quad c<\left(\beta a_{k+1}\right)^{r-t} .
$$

Since $a_{k} \geq a_{k+1}$ and $r>t$, this is impossible. Hence (13) holds. If $n \geq k+2$, we need to verify that $x_{j}^{\prime} \geq x_{j}$ for $k+2 \leq j \leq n$. If not, there exists $s \geq k+2$ such that $x_{s}>x_{s}^{\prime}$. Since $x_{k+1}^{\prime}>x_{k+1}$ by (13), again by (12) we have

$$
c<\left(\beta a_{s}\right)^{r-t} \quad \text { and } \quad c<\left(\beta a_{k+1}\right)^{r-t}
$$


which will imply that $a_{s}>a_{k+1}$, a contradiction with the arrangement of the $a_{j}$ 's. This completes the proof.

Theorem 2.8 (cf. [4]). Let $\mathbf{a}, \mathbf{w} \in \mathbf{R}_{+}^{n}$.

(a) Suppose $r_{1}>t_{1}, r_{2}>t_{2} \geq t_{1} \geq 0$, and $t_{2}-t_{1} \leq r_{2}-r_{1}$. Then

$$
B_{n}^{r_{1}, t_{1}}(\mathbf{a} ; \mathbf{w}) \leq B_{n}^{r_{2}, t_{2}}(\mathbf{a} ; \mathbf{w}) .
$$

(b) For $1 \leq t<r$,

$$
H_{n}^{[t]}(\mathbf{a} ; \mathbf{w}) \leq H_{n}^{[r]}(\mathbf{a} ; \mathbf{w})
$$

(c) For $1<r$,

$$
M_{n}^{[r]}(\mathbf{a} ; \mathbf{w}) \leq H_{n}^{[r]}(\mathbf{a} ; \mathbf{w}) .
$$

Equality holds in (a) (resp. (b), (c)) if and only if $a_{1}=a_{2}=\cdots=a_{n}$.

Proof. For $\mathbf{a} \in \mathbf{R}_{+}^{n}$, we may assume that $a_{1} \geq a_{2} \geq \cdots \geq a_{n}$ (by rearranging the subscripts if necessary). Set $f_{i}(\mathbf{a})=a_{i}, 1 \leq i \leq n$.

(a) Define $x_{j}^{\prime}=a_{j}^{t_{1}} / \sum_{i=1}^{n} w_{i} a_{i}^{t_{1}}$, and $x_{j}=a_{j}^{r_{1}} / \sum_{i=1}^{n} w_{i} a_{i}^{r_{1}}, 1 \leq j \leq n$, then $\mathbf{x} \succ \mathbf{x}^{\prime}$ by Lemma 2.7. Hence by Theorem 2.5

$$
\begin{aligned}
B_{n}^{r_{1}, t_{1}}(\mathbf{a} ; \mathbf{w}) & =L_{n, n}^{\left[r_{1}-t_{1}\right]}\left[\mathbf{f} ; \mathbf{x}^{\prime} ; \mathbf{w}\right](\mathbf{a}) \\
& \leq L_{n, n}^{\left[r_{1}-t_{1}\right]}[\mathbf{f} ; \mathbf{x} ; \mathbf{w}](\mathbf{a}) \\
& \leq L_{n, n}^{\left[r_{2}-t_{2}\right]}[\mathbf{f} ; \mathbf{x} ; \mathbf{w}](\mathbf{a}) \\
& =B_{n}^{r_{2}, t_{2}}(\mathbf{a} ; \mathbf{w}) .
\end{aligned}
$$

(b) Set $x_{j}^{\prime}=a_{j}^{t-1} / \sum_{i=1}^{n} w_{i} a_{i}^{t-1}$ and $x_{j}=a_{j}^{r-1} / \sum_{i=1}^{n} w_{i} a_{i}^{r-1}, 1 \leq i \leq n$, then $\mathbf{x} \succ \mathbf{x}^{\prime}$ by Lemma 2.7 and

$$
H_{n}^{[t]}(\mathbf{a} ; \mathbf{w})=L_{n, n}^{[1]}\left[\mathbf{f} ; \mathbf{x}^{\prime} ; \mathbf{w}\right](\mathbf{a}) \leq L_{n, n}^{[1]}[\mathbf{f} ; \mathbf{x} ; \mathbf{w}](\mathbf{a})=H_{n}^{[r]}(\mathbf{a} ; \mathbf{w}) .
$$

Since $H_{n}^{[t]}(\mathbf{a} ; \mathbf{w})=B_{n}^{t, t-1}(\mathbf{a} ; \mathbf{w})$, this inequality is also a corollary to (a).

(c) Take $x_{j}^{\prime}=1 / w_{+}$, and $x_{j}=a_{j} / \sum_{i=1}^{n} w_{i} a_{i}^{r}, 1 \leq j \leq n$. Clearly $\mathbf{x} \succ \mathbf{x}^{\prime}$ and so

$$
M_{n}^{[r]}(\mathbf{a} ; \mathbf{w})=L_{n, n}^{[r]}\left[\mathbf{f} ; \mathbf{x}^{\prime} ; \mathbf{w}\right](\mathbf{a}) \leq L_{n, n}^{[r]}[\mathbf{f} ; \mathbf{x} ; \mathbf{w}](\mathbf{a})=H_{n}^{[r]}(\mathbf{a} ; \mathbf{w}) .
$$

For equality, since the proofs are similar, we shall only give the proof of (a). We need to show that the condition $\operatorname{EQ}\left(\mathbf{f} ; \mathbf{x} \succ \mathbf{x}^{\prime} ; \mathbf{a}\right)$ is satisfied. To confirm this, it suffices to verify that if the $a_{j}$ 's are not all equal, then there exists an $i, i \neq k$, so that $f_{i}(\mathbf{a}) \neq f_{k}(\mathbf{a})$, where $x_{k+1}^{\prime}>x_{k+1}$ since (12) and (13) still hold. Hence by (12) $c>\left(\beta a_{k+1}\right)^{r_{1}-t_{1}}$. We shall complete the proof by verifying that $f_{k+1}(\mathbf{a}) \neq f_{k}(\mathbf{a})$, otherwise, $a_{k}=f_{k}(\mathbf{a})=f_{k+1}(\mathbf{a})=a_{k+1}$. Hence, $c>\left(\beta a_{k}\right)^{r_{1}-t_{1}}$, again, by (12), $x_{k}^{\prime}>x_{k}$ which contradicts (13).

\section{INTERPOLATION OF INEQUALITIES}

In this section, we shall apply Theorem 2.5 (b) to refine many well-known inequalities including an inequality of Ky Fan ([12]). We adopt the same notations introduced in sections 1 and 2 unless otherwise specified. For simplicity of the statements and the intuition of the arguments, we will focus on the interpolation of double inequalities with three quantities. The general case of interpolating a chain of any finite number of inequalities is similar. 
As a special case of Definition 2.3 , let $m=3, \triangle(\mathbf{w})$ be the triangle in $\mathbf{R}^{3}$ with any fixed $\mathbf{w} \in \mathbf{R}_{+}^{3}$, and

$$
f_{1}(\mathbf{a})=A_{n}(\mathbf{a}), \quad f_{2}(\mathbf{a})=G_{n}(\mathbf{a}), \quad f_{3}(\mathbf{a})=H_{n}(\mathbf{a}) .
$$

For a fixed $r \geq 0$, define a 3 -variable function $\Phi(\mathbf{x})$ of $\mathbf{x}=\left(x_{1}, x_{2}, x_{3}\right)$ on $\triangle(\mathbf{w})$ via Definition 2.3 as

$$
\Phi(\mathbf{x})(\mathbf{a})=L_{n, 3}^{[r]}[\mathbf{f} ; \mathbf{x} ; \mathbf{w}](\mathbf{a})
$$

Then it is clear that,

$$
\Phi\left(\mathbf{W}_{1}\right)(\mathbf{a})=A_{n}(\mathbf{a}), \quad \Phi\left(\mathbf{W}_{2}\right)(\mathbf{a})=G_{n}(\mathbf{a}), \quad \Phi\left(\mathbf{W}_{3}\right)(\mathbf{a})=H_{n}(\mathbf{a}) .
$$

From Theorem 2.5 (b), we have

Theorem 3.1. If $\mathbf{x}, \mathbf{x}^{\prime} \in \triangle(\mathbf{w}), \mathbf{w} \in \mathbf{R}_{+}^{n}$, and $\mathbf{x} \succ \mathbf{x}^{\prime}, \mathbf{x} \neq \mathbf{x}^{\prime}$, then

$$
\Phi(\mathbf{x})(\mathbf{a}) \geq \Phi\left(\mathbf{x}^{\prime}\right)(\mathbf{a})
$$

with equality holds if and only if $a_{1}=a_{2}=\cdots=a_{n}$.

For each $\mathbf{w} \in \mathbf{R}_{+}^{3}$, since $\sum_{i=1}^{3} w_{i} x_{i}=1, \Phi(\mathbf{x})$ provides a two-parameter continuous family of means for $a_{1}, a_{2}, \cdots, a_{n}$ with $A_{n}(\mathbf{a})$ as the largest member and $H_{n}(\mathbf{a})$ as the smallest.

By applying the same argument as we used in Theorem 3.1, we are able to refine the following well-known inequality of Ky Fan which has been the subject of a number of articles $([1,2,3,10,19,20])$ :

$$
\frac{H_{n}(\mathbf{a})}{H_{n}^{\prime}(\mathbf{a})} \leq \frac{G_{n}(\mathbf{a})}{G_{n}^{\prime}(\mathbf{a})} \leq \frac{A_{n}(\mathbf{a})}{A_{n}^{\prime}(\mathbf{a})}
$$

where $A_{n}^{\prime}(\mathbf{a}), G_{n}^{\prime}(\mathbf{a})$ and $H_{n}^{\prime}(\mathbf{a})$ are the arithmetic mean, geometric mean, and harmonic mean of $1-a_{1}, \cdots, 1-a_{n}$, respectively, when $a_{i} \in(0,1 / 2], i=1,2, \cdots, n$.

Recently, Yang and Wang have introduced two continuous monotonic functions $p(t)(\mathbf{a})$ and $q(t)(\mathbf{a})$ such that $([20])$

$$
\frac{H_{n}(\mathbf{a})}{H_{n}^{\prime}(\mathbf{a})} \leq p(t)(\mathbf{a}) \leq \frac{G_{n}(\mathbf{a})}{G_{n}^{\prime}(\mathbf{a})} \leq q(t)(\mathbf{a}) \leq \frac{A_{n}(\mathbf{a})}{A_{n}^{\prime}(\mathbf{a})}
$$

with $p(1 / n)=H_{n}(\mathbf{a}) / H_{n}^{\prime}(\mathbf{a}), p(0)=G_{n}(\mathbf{a}) / G_{n}^{\prime}(\mathbf{a})=q(0)$, and $q(1 / n)=$ $A_{n}(\mathbf{a}) / A_{n}^{\prime}(\mathbf{a})$.

Now, assume that $\triangle(\mathbf{w})$ is the same as in Theorem 3.1. Let

$$
f_{1}(\mathbf{a})=\frac{A_{n}(\mathbf{a})}{A_{n}^{\prime}(\mathbf{a})}, \quad f_{2}(\mathbf{a})=\frac{G_{n}(\mathbf{a})}{G_{n}^{\prime}(\mathbf{a})}, \quad f_{3}(\mathbf{a})=\frac{H_{n}(\mathbf{a})}{H_{n}^{\prime}(\mathbf{a})},
$$

and for a fixed $r \geq 0$, let

$$
\Psi(\mathbf{x})(\mathbf{a})=L_{n, 3}^{[r]}[\mathbf{f} ; \mathbf{x} ; \mathbf{w}](\mathbf{a})
$$

Then we have

$$
\Psi\left(\mathbf{W}_{1}\right)(\mathbf{a})=\frac{A_{n}(\mathbf{a})}{A_{n}^{\prime}(\mathbf{a})}, \quad \Psi\left(\mathbf{W}_{2}\right)(\mathbf{a})=\frac{G_{n}(\mathbf{a})}{G_{n}^{\prime}(\mathbf{a})}, \quad \text { and } \quad \Psi\left(\mathbf{W}_{3}\right)(\mathbf{a})=\frac{H_{n}(\mathbf{a})}{H_{n}^{\prime}(\mathbf{a})} .
$$

We obtain the following counterpart of Theorem 3.1 which is simply another refinement of Fan's inequality in terms of a two-parameter family of inequalities.

Theorem 3.1'. For $\mathbf{w} \in \mathbf{R}_{+}^{3}, \mathbf{x}, \mathbf{x}^{\prime} \in \triangle(\mathbf{w}), \mathbf{x} \succ \mathbf{x}^{\prime}$ and $\mathbf{x} \neq \mathbf{x}^{\prime}$, then

$$
\Psi(\mathbf{x})(\mathbf{a}) \geq \Psi\left(\mathbf{x}^{\prime}\right)(\mathbf{a})
$$

with equality if and only if $a_{1}=\cdots=a_{n}$. 


\section{Concluding Remarks}

The original inequality of Ky Fan was

$$
\frac{G_{n}(\mathbf{a})}{G_{n}^{\prime}(\mathbf{a})} \leq \frac{A_{n}(\mathbf{a})}{A_{n}^{\prime}(\mathbf{a})} .
$$

Later on, many articles developed it into the form of (15) with various techniques. As a matter of fact, since there are many symmetric means $P_{n}^{[r]}(\mathbf{a}), r=1, \cdots, n$, lying between $A_{n}(\mathbf{a})$ and $G_{n}(\mathbf{a})$, and $P_{n}^{[-r]}(\mathbf{a}), r=1, \cdots, n$, lying between $G_{n}(\mathbf{a})$ and $H_{n}(\mathbf{a})$, the following more general inequalities of Fan-type are also valid [12, p. 32]:

$$
\begin{aligned}
\frac{H_{n}(\mathbf{a})}{H_{n}^{\prime}(\mathbf{a})} & =\frac{P_{n}^{[-1]}(\mathbf{a})}{P_{n}^{[-1]^{\prime}}(\mathbf{a})} \leq \frac{P_{n}^{[-2]}(\mathbf{a})}{P_{n}^{[-2]^{\prime}}(\mathbf{a})} \leq \cdots \leq \frac{P_{n}^{[-n+1]}(\mathbf{a})}{P_{n}^{[-n+1]^{\prime}}(\mathbf{a})} \leq \frac{P_{n}^{[-n]}(\mathbf{a})}{P_{n}^{[-n]^{\prime}}(\mathbf{a})}=\frac{G_{n}(\mathbf{a})}{G_{n}^{\prime}(\mathbf{a})} \\
& =\frac{P_{n}^{[n]}(\mathbf{a})}{P_{n}^{[n]^{\prime}}(\mathbf{a})} \leq \frac{P_{n}^{[n-1]}(\mathbf{a})}{P_{n}^{[n-1]^{\prime}}(\mathbf{a})} \leq \cdots \leq \frac{P_{n}^{[2]}(\mathbf{a})}{P_{n}^{[2]^{\prime}}(\mathbf{a})} \leq \frac{P_{n}^{[1]}(\mathbf{a})}{P_{n}^{[1]^{\prime}}(\mathbf{a})}=\frac{A_{n}(\mathbf{a})}{A_{n}^{\prime}(\mathbf{a})}
\end{aligned}
$$

where every quantity with a prime in the denominator represents the corresponding mean of $1-a_{1}, \cdots, 1-a_{n}$, for $a_{i} \in(0,1 / 2], i=1,2, \cdots, n$.

We may use a $2(n-1)$-simplex $\triangle(\mathbf{w})$ in $\mathbf{R}^{2 n-1}$ as described in section 2 , in the same spirit of Theorems $3.1,3.1^{\prime}$ to generalize the chain of inequalities of Fan-type above, or the chain of symmetric mean inequalities in section 1 to a $(2 n-2)$ parameter family of inequalities. More specifically, Let $\triangle(\mathbf{w})$ be the $2(n-1)$ simplex in $\mathbf{R}^{2 n-1}$ with vertices $\mathbf{W}_{i}$ as defined in section 2 , where $\mathbf{w} \in \mathbf{R}_{+}^{2 n-1}$. Set

$$
f_{i}(\mathbf{a})=P_{n}^{[i]}(\mathbf{a}), 1 \leq i \leq n ; \text { and } f_{i+n}(\mathbf{a})=P_{n}^{[-i]}(\mathbf{a}), 1 \leq i \leq n-1 .
$$

Then we can view $\triangle(\mathbf{w})$ as an index domain for $\Phi(\mathbf{x})$ whose special values on the vertices are symmetric means, and for $\Psi(\mathbf{x})$ whose special values on the vertices are the quotients of symmetric means in the inequality of Fan-type. In contrast with [9], we may call the functions $\Phi(\mathbf{x})(\mathbf{a})$ and $\Psi(\mathbf{x})(\mathbf{a})$ multi-variable extended mean values of $a_{1}, \cdots, a_{n}$ and multi-variable extended mean value quotients of Fan-type. In [9], Leach and Sholander demonstrated some very interesting properties of multivariable extended mean values. Likewise, one could carry out a similar discussion for our generalized power means.

Means and their inequalities have always been an important resource for geometric extremum problems. In his wonderful book Induction and Analogy in Mathematics [16], Pólya pointed out the remarkable analogy between the arithmetic mean-geometric mean inequality and the classical isoperimetric inequality for a simple closed plane curve. Therefore, any refinement of one object will likely imply a similar refinement of the other. In Ivan Niven's book Maxima and Minima Without Calculus [14] that has enlightened many interesting articles in MAA journals, inequalities involving different means are key ingredients. In fact, geometric inequalities which are derived from inequalities of means are collected in many books such as $[6,13,14,16,17]$ etc. Regarding $\mathbf{a}=\left(a_{1}, a_{2}, \cdots, a_{n}\right)$ as the side lengths of an $n$-sided plane polygon $\mathcal{P}_{n}$, in [7], we have used some refined inequalities of means to establish many geometric inequalities of isoperimetric type for polygons. In [13], one can find many geometric inequalities for an $n$-simplex $\mathcal{S}_{n}$ in $\mathbf{R}^{m}$ where $\mathbf{a}=\left(a_{1}, a_{2}, \cdots, a_{n}\right)$ represents a collection of the areas of its $n$ faces, or a collection of its $n$ altitudes, and so on, as immediate consequences of some inequalities in 
means. The method of interpolating inequalities is certainly applicable to refining geometric inequalities. We have used the results of this paper and obtained some new interesting geometric inequalities in [8].

\section{ACKNOWLEDGEMENT}

The authors are grateful to the referee for his suggestions that improved the presentation of this paper.

\section{REFERENCES}

[1] H. Alzer, On an inequality of Ky Fan, J. Math. Anal. Appl., 137 (1989), 168-172. MR 90a:26029

[2] H. Alzer, Inequalities for Arithmetic, Geometric and Harmonic means, Bull. London Math. Soc., 22 (1990), 362-366. MR 91g:26026

[3] E. F. Beckenbach and R. Bellman, Inequalities, Springer-Verlag, New York, 1965. MR 33:236

[4] P. S. Bullen, D. S. Mitrinović and P. M. Vasić, Means and Their Inequalities, Reidel, Dordrecht, 1988. MR 89d:26003

[5] G. Hardy, J. E. Littlewood and G. Pólya Inequalities, Cambridge Univ. Press, Cambridge, New York, 1951.

[6] M. D. Kazarinoff, Geometric Inequalities, New Math. Library, MAA, 1961. MR 24:A1

[7] H. T. Ku, M. C. Ku and X. M. Zhang, Analytic and Geometric Isoperimetric Inequalities, Journal of Geometry, 53 (1995), 100-121. MR 96f:52012

[8] H. T. Ku, M. C. Ku and X. M. Zhang, Inequalities for Symmetric Means, Symmetric Harmonic Means and Their Applications, Bull. Austral. Math. Soc. 56 (1997), 409-420. CMP 98:06

[9] E. B. Leach and M. C. Sholander, Multi-variable Extended Mean Values, J. Math. Anal. Appl., 104 (1984), 390-407. MR 86b:26033

[10] M. T. McGregor, On Some Inequalities of Ky Fan and Wang-Wang, J. Math. Anal. Appl., 180 (1993), 182-188. MR 94i:26012

[11] D. S. Mitrinović, Analytic Inequalities, Springer-Verlag, New York, 1970. MR 43:448

[12] D. S. Mitrinović, J. E. Pečarić and A. M. Fink, Classical and New Inequalities in Analysis, Kluwer Academic Publishers, Dordrecht/Boston/London, 1993. MR 94c:00004

[13] D. S. Mitrinović, J. E. Pečarić and V. Volenec, Recent Advances in Geometric Inequalities, Kluwer Academic Publishers, Dordrecht/Boston/London, 1989. MR 91k:52014

[14] I. Niven, Maxima and Minima Without Calculus, The Mathematical Association of America, 1981. MR 83i:52011

[15] R. Osserman, The Isoperimetric Inequalities, Bull. Amer. Math. Soc. 84 (1978), 1182-1238. MR 58:18161

[16] G. Pólya, Mathematics and Plausible Reasoning, Vol I., Induction and Analogy in Mathematics, Princeton, 1954. MR 92a:00028a

[17] G. Pólya and G. Szegö, Isoperimetric Inequalities in Mathematical Physics, Ann. of Math., studies No. 27, Princeton Univ., Princeton, 1951. MR 13:270d

[18] G. Russell, Connected Means, Math. Gazette, Vol. 18, No. 460 (1988), 97-100.

[19] W. L. Wang and P. F. Wang, A Class of Inequalities for the Symmetric Functions, Acta Math. Sinica (Chinese), 27 (1984), No.4, 485-497. MR 96h:05022

[20] G. S. Yang and C. S. Wang, Refinements on an Inequality of Ky Fan, J. Math. Anal. Appl., 201 (1996), 955-965. MR 97e:26023

Department of Mathematics and Statistics, University of Massachusetts, Amherst, MASSACHUSETTS 01003

E-mail address: meiku@math.umass.edu

Department of Mathematics and Statistics, University of South Alabama, Mobile, Alabama 36688

E-mail address: zhang@mathstat.usouthal.edu 